\title{
Comparison of Lipoprotein-Associated Phospholipase A2 and High Sensitive C-Reactive Protein as Determinants of Metabolic Syndrome in Subjects without Coronary Heart Disease: In Search of the Best Predictor
}

\author{
Mónica Acevedo, ${ }^{1}$ Paola Varleta, ${ }^{2}$ Verónica Kramer, ${ }^{1}$ \\ Giovanna Valentino, ${ }^{1}$ Teresa Quiroga, ${ }^{3}$ Carolina Prieto, ${ }^{4}$ Jacqueline Parada, ${ }^{3}$ \\ Marcela Adasme, ${ }^{1}$ Luisa Briones, ${ }^{2}$ and Carlos Navarrete ${ }^{5}$ \\ ${ }^{1}$ División de Enfermedades Cardiovasculares, Escuela de Medicina, Facultad de Medicina, Pontificia Universidad Católica de Chile, \\ Marcoleta 367, Octavo Piso, Santiago Centro, 8330024 Santiago, Chile \\ ${ }^{2}$ Unidad de Prevención ACV y Rehabilitación Cardiaca, División de Cardiología y Cirugía Cardiaca, Hospital de la Dirección de \\ Previsión de Carabineros de Chile, Vital Apoquindo 1200, 2Piso, Las Condes, 7601003 Santiago, Chile \\ ${ }^{3}$ Departamento de Laboratorios Clínicos, Escuela de Medicina, Facultad de Medicina, Pontificia Universidad Católica de Chile, \\ Avenida Vicuña Mackenna 4686, Macul, 7820436 Santiago, Chile \\ ${ }^{4}$ Laboratorio Clínico, Hospital de la Dirección de Previsión de Carabineros de Chile, Vital Apoquindo 1200, 2Piso, Las Condes, \\ 7601003 Santiago, Chile \\ ${ }^{5}$ Departamento de Matemáticas, Facultad de Ciencias, Universidad de la Serena, Avenida Raúl Bitrán Nachary s/n, \\ 1700000 La Serena, Chile
}

Correspondence should be addressed to Mónica Acevedo; macevedo@med.puc.cl and Paola Varleta; pvarleta@manquehue.net

Received 3 September 2014; Accepted 21 October 2014

Academic Editor: Ilias Migdalis

Copyright (C) 2015 Mónica Acevedo et al. This is an open access article distributed under the Creative Commons Attribution License, which permits unrestricted use, distribution, and reproduction in any medium, provided the original work is properly cited.

High sensitivity C-reactive protein (hsCRP) is a marker of metabolic syndrome (MS) and cardiovascular (CV) disease. Lipoproteinassociated phospholipase A2 (Lp-PLA2) also predicts CV disease. There are no reports comparing these markers as predictors of MS. Methods. Cross-sectional study comparing Lp-PLA2 and hsCRP as predictors of MS in asymptomatic subjects was carried out; 152 subjects without known atherosclerosis participated. Data were collected on demographics, cardiovascular risk factors, anthropometric and biochemical measurements, and hsCRP and Lp-PLA2 activity levels. A logistic regression analysis was performed with each biomarker and receiver operating characteristic (ROC) curves were constructed for MS. Results. Mean age was $46 \pm 11$ years, and $38 \%$ of the subjects had MS. Mean Lp-PLA2 activity was $185 \pm 48 \mathrm{nmol} / \mathrm{mL} / \mathrm{min}$, and mean hsCRP was 2.1 $\pm 2.2 \mathrm{mg} / \mathrm{L}$. Subjects with MS had significantly higher levels of Lp-PLA2 $(P=0.03)$ and hsCRP $(P<0.0001)$ than those without MS. ROC curves showed that both markers predicted MS. Conclusion. Lp-PLA2 and hsCRP are elevated in subjects with MS. Both biomarkers were independent and significant predictors for MS, emphasizing the role of inflammation in MS. Further research is necessary to determine if inflammation predicts a higher risk for CV events in MS subjects.

\section{Introduction}

Metabolic syndrome (MS) is a group of cardiovascular (CV) risk factors including abdominal obesity, hypertriglyceridemia, low high-density lipoprotein cholesterol (HDL-C), dysglycemia, and high blood pressure (BP) [1]. This condition is associated with a proinflammatory and prothrombotic condition [2]. Numerous studies have shown that a significant increase in systemic inflammatory markers, such as high sensitivity C-reactive protein (hsCRP), interleukin-6, and tumor necrosis factor- $\alpha$ (TNF- $\alpha$ ), among others, is observed in subjects with MS [3]. 
High sensitivity C-reactive protein is a good predictor of $\mathrm{MS}$ and is strongly associated with abdominal obesity [4]. Moreover, this biomarker predicts CV events in healthy people as well as in those with atherosclerotic disease $[5,6]$. It has been reported that hsCRP is elevated in an urban population of Santiago, Chile, with MS, and there is a clear correlation between MS, hsCRP, and subclinical atherosclerosis in this population [7]. Thus, the clustering of CV risk factors and inflammation observed in MS increase the risk of atherosclerosis.

Lipoprotein-associated phospholipase A2 (Lp-PLA2) is a recently described inflammatory marker [8]. It is an enzyme produced by macrophages that hydrolyzes phospholipids of oxidized low-density lipoprotein (LDL), releasing oxidized fatty acids and lysophosphatidylcholine, which are potent proinflammatory and prooxidative molecules [8]. Since oxidized LDL is mostly located in the intima of the artery, the products of Lp-PLA2 activity are generated mainly in the vascular wall. Therefore, these mediators play an important role in inflammation at the vascular level. Given that atherosclerosis is an inflammatory disease which begins in the vascular wall, Lp-PLA2 may have a prominent role in its pathophysiology [8].

Both Lp-PLA2 and hsCRP are associated with coronary and cerebrovascular atherosclerotic disease in populations with and without a history of CV events [9-12]. Furthermore, Lp-PLA2 is related to CV risk factors, with a strong correlation with LDL, as well as various components of MS (e.g., abdominal obesity) [13]. The vast majority of published studies do not show any association between LpPLA2 and hsCRP; hence, these inflammatory markers have been considered to be independent of each other. There are no reports in literature that compare these markers as predictors of MS.

The objective of this study was to analyze and compare the levels of Lp-PLA2 and hsCRP as predictors of MS in subjects without atherosclerotic disease.

\section{Materials and Methods}

This was a descriptive cross-sectional study in 152 subjects (69 women), without history of atherosclerotic disease, who were recruited in two preventive cardiology centers of Santiago between October 2011 and June 2012: Hospital Clínico de la Pontificia Universidad Católica de Chile and Hospital de la Previsión de Carabineros de Chile. Subjects between 18 and 70 years old were included. Exclusion criteria were (a) history of coronary heart disease and/or carotid or peripheral vascular disease; (b) lipid-lowering therapy (statins, ezetimibe, fibrates, nicotinic acid, and omega 3); (c) use of oral contraceptives or hormone replacement therapy; (d) chronic intake of anti-inflammatory medicines (steroidal and nonsteroidal); (e) acute and/or chronic inflammatory diseases; and (f) pregnancy.

The investigation protocol was approved by the ethics committees of both institutions. Subjects were contacted by telephone and invited to participate by nurses or doctors in charge of the study.
After signing the written, informed consent, patients were subjected to clinical data collection and anthropometric and laboratory measurements, all detailed below.

2.1. Data Collection. During the visit to the centers, subjects were interviewed about demographics, medical history, education, physical activity, family medical history, and intake of medications.

2.1.1. Anthropometric and Laboratory Measures. Weight, height, body mass index (BMI), and hip and waist circumferences were measured. The latter was measured in the midpoint between the last rib and the iliac crest. Blood pressure was measured following the recommendations of the Seventh Joint National Committee [14], with the subject sitting and after 5 minutes of rest.

Venous blood samples were taken after 12-hour fasting for determining the following biochemical parameters:

(i) Lp-PLA2: samples for analysis of Lp-PLA2 activity were frozen and stored at $-70^{\circ} \mathrm{C}$. Levels of $\mathrm{Lp}$ PLA2 activity were determined by enzymatic method (DIADEXUS, USA), Cobas 8000 analyzer, c702 module. Prior to the analysis, precision and trueness of the enzymatic test were assessed locally, and a calibration curve was constructed, which was sent to the international center for approval. Following the approval, the analysis of the subjects' samples was performed.

(ii) Total cholesterol (total-C): colorimetric enzymatic method, Roche Diagnostics Cobas analyzer Cobas 8000, c702 module.

(iii) HDL-C: colorimetric homogeneous enzymatic method, Roche Diagnostics Cobas, Cobas 8000 analyzer, c702 module.

(iv) Triglycerides: white colorimetric enzymatic method with glycerol, Roche Diagnostics Cobas, Cobas 8000 analyzer, c702 module.

(v) LDL cholesterol (LDL-C): calculated by Friedewald formula.

(vi) Blood glucose: enzymatic method (hexokinase), Roche Diagnostics Cobas, Cobas 8000 analyzer, c702 module.

(vii) Creatinine: Jaffé method, Roche Diagnostics Cobas, Cobas 8000 analyzer, c70 module.

(viii) hsCRP: nephelometric method on BN ProSpec analyzer, Siemens (detection limit $0.16 \mathrm{mg} / \mathrm{L}$ ).

(ix) Fibrinogen: Clauss method in ACL Top 500 analyzer, Instrumentation Laboratory.

2.2. Variables. Hypertensives were subjects who had a prior diagnosis according to the JNC 7 [14], with or without pharmacologic therapy, and/or those with an average blood pressure $\geq 140 / 90 \mathrm{~mm} \mathrm{Hg}$. Dyslipidemics were subjects who had LDL-C level $\geq 130 \mathrm{mg} / \mathrm{dL}$, HDL-C level $<40 \mathrm{mg} / \mathrm{dL}$ in men, $<50 \mathrm{mg} / \mathrm{dL}$ in women, or non-HDL-C level $\geq 160 \mathrm{mg} / \mathrm{dL}$ 
TABLE 1: Demographic data and prevalence of traditional cardiovascular risk factors in the total sample divided by gender.

\begin{tabular}{lcccc}
\hline & $\begin{array}{c}\text { Total } \\
(n=152)\end{array}$ & $\begin{array}{c}\text { Men } \\
(n=83)\end{array}$ & $\begin{array}{c}\text { Women } \\
(n=69)\end{array}$ & $P$ \\
\hline Age (years) & $46 \pm 11$ & $45 \pm 11$ & $47 \pm 11$ & NS \\
Educational level (years) & $13 \pm 3$ & $14 \pm 2$ & $13 \pm 4$ & NS \\
Dyslipidemia (\%) & 62 & 68 & 55 & NS \\
Hypertension (\%) & 30 & 31 & 29 & NS \\
Smoking (\%) & 31 & 31 & 30 & NS \\
Diabetes (\%) & 5 & 7 & 3 & NS \\
Physical inactivity (\%) & 78 & 76 & 80 & NS \\
Overweight (\%) & 37 & 41 & 33 & NS \\
Obesity (\%) & 33 & 34 & 32 & NS \\
Family history of CHD (\%) & 13 & 10 & 16 & NS \\
\hline Data a presented as m & & & &
\end{tabular}

Data are presented as mean \pm SD or percentage.

CHD: coronary heart disease; NS: not significant.

in the laboratory assessment. Subjects were considered diabetics if they had a prior diagnosis, with or without drug treatment, and/or if they had a fasting blood glucose $\geq 126 \mathrm{mg} / \mathrm{dL}$ during the study, according to the American Diabetes Association criteria. Current smokers were those subjects who smoked daily during the last month, and exsmokers were those subjects who had at least six consecutive months without smoking. The recent harmonized criteria were used for the diagnosis of MS, which includes a waist circumference $\geq 90 \mathrm{~cm}$ in men and $\geq 80 \mathrm{~cm}$ in women for Latin American populations [1].

2.3. Statistical Analysis. The software R 2.15 .2 was used for statistical analysis. A logistic regression analysis was performed with each biomarker, adjusted for age and gender. Smooth receiver operating characteristic (ROC) curves were constructed with cubic splines for MS (area under the curve $C$ value $=0.50$ implies a predictive value equivalent to chance). Comparisons of means (expressed as mean \pm standard deviation [SD]) are based on the analysis of variance and linear regression models.

\section{Results}

This study included 152 subjects (45\% women) with a mean age of $46 \pm 11$ years. Table 1 shows demographic data and prevalence for CV risk factors. Prevalence rate for hypertension, diabetes, dyslipidemia, and current smoking was $30 \%, 5 \%, 62 \%$, and $31 \%$, respectively, with no significant differences observed between genders. Prevalence of MS was $38 \%$ (57 subjects) in the total sample with no significant differences between genders: $42 \%$ and $32 \%$ in men and women, respectively. Table 2 provides anthropometric and laboratory measures from all subjects divided by gender. Men had significantly higher waist circumference $(P<0.01)$, systolic and diastolic BP $(P<0.0001)$, and creatinine levels $(P<0.0001)$ than women. Women had higher HDL-C levels $(P<0.0001)$.
TABLE 2: Anthropometric measurements and laboratory data for all subjects divided by gender.

\begin{tabular}{lcccc}
\hline & $\begin{array}{c}\text { Total } \\
(n=152)\end{array}$ & $\begin{array}{c}\text { Men } \\
(n=83)\end{array}$ & $\begin{array}{c}\text { Women } \\
(n=69)\end{array}$ & $P$ \\
\hline $\begin{array}{l}\text { BMI } \\
\text { Waist circumference }\end{array}$ & $93 \pm 4$ & $28 \pm 4$ & $28 \pm 5$ & NS \\
(cm) & $95 \pm 10$ & $90 \pm 11$ & $<0.01$ \\
Waist $\geq 90 / 80 \mathrm{~cm}$ & $115(76 \%)$ & $61(74 \%)$ & $54(79 \%)$ & NS \\
(men/women), $n(\%)$ & $119 \pm 15$ & $124 \pm 14$ & $113 \pm 16$ & $<0.0001$ \\
SBP (mm Hg) & $76 \pm 13$ & $81 \pm 11$ & $70 \pm 13$ & $<0.0001$ \\
DBP (mm Hg) & $91 \pm 24$ & $93 \pm 29$ & $89 \pm 16$ & NS \\
$\begin{array}{l}\text { Blood glucose } \\
\text { (mg/dL) }\end{array}$ & $208 \pm 44$ & $209 \pm 47$ & $207 \pm 39$ & NS \\
Total-C (mg/dL) & $52 \pm 15$ & $46 \pm 13$ & $58 \pm 14$ & $<0.0001$ \\
HDL-C (mg/dL) & $128 \pm 36$ & $131 \pm 38$ & $126 \pm 34$ & NS \\
LDL-C (mg/dL) & $154 \pm 43$ & $158 \pm 43$ & $148 \pm 42$ & NS \\
$\begin{array}{l}\text { Non-HDL-C (mg/dL) } \\
\text { Creatinine (mg/dL) }\end{array}$ & $0.8 \pm 0.2$ & $0.9 \pm 0.1$ & $0.7 \pm 0.1$ & $<0.0001$ \\
hsCRP (mg/L) & $2.1 \pm 2.2$ & $2.2 \pm 2.2$ & $2.0 \pm 2.2$ & NS \\
$\begin{array}{l}\text { Lp-PLA2 } \\
\text { (nmol/mL/min) }\end{array}$ & $185 \pm 48$ & $201 \pm 49$ & $166 \pm 38$ & $<0.0001$ \\
\hline
\end{tabular}

Values expressed as mean \pm SD, except where indicated.

BMI: body mass index; DBP: diastolic blood pressure; HDL-C: high-density lipoprotein cholesterol; hsCRP: high sensitive C-reactive protein; LDL-C: low-density lipoprotein cholesterol; Lp-PLA2: lipoprotein-associated phospholipase A2; NS: not significant; SBP: systolic blood pressure; SD: standard deviation; total-C: total cholesterol.

Mean level of hsCRP was $2.1 \pm 2.2 \mathrm{mg} / \mathrm{L}$, and there were no significant differences between men and women (Table 2). Likewise, there were no significant differences in the prevalence of high levels of hsCRP (defined as $>2 \mathrm{mg} / \mathrm{L}$ ) between genders (39\% in men and 36\% in women). Of note, hsCRP was significantly and directly correlated with BMI, waist circumference, blood glucose, systolic BP, non-HDL$\mathrm{C}$, and fibrinogen, and it was inversely and significantly correlated with HDL-C (Table 3).

Mean level of Lp-PLA2 activity was $185 \pm 48 \mathrm{nmol} / \mathrm{mL} /$ min in the total sample with significant differences between men and women: $201 \mathrm{nmol} / \mathrm{mL} / \mathrm{min}$ and $166 \mathrm{nmol} / \mathrm{mL} / \mathrm{min}$ $(P<0.0001)$, respectively (Table 2$)$. Lp-PLA2 was significantly and directly correlated with BMI, waist circumference, diastolic BP, LDL-C, non-HDL-C, and plasma creatinine; HDL-C was inversely and significantly correlated with LpPLA2. No significant correlations were found between LpPLA2 and blood glucose, systolic BP, hsCRP, and fibrinogen (Table 3). No differences were found between Lp-PLA2 activity of active smokers and nonsmokers.

As shown in Table 4, both Lp-PLA2 and hsCRP were significantly higher in subjects with MS than in those without MS. In subjects with MS, Lp-PLA2 was $198 \pm 45 \mathrm{nmol} / \mathrm{mL} /$ min versus $180 \pm 48 \mathrm{nmol} / \mathrm{mL} / \mathrm{min}$ in subjects without MS $(P=0.03)$. Similarly, hsCRP was $4.1 \pm 3.3 \mathrm{mg} / \mathrm{L}$ in subjects with MS versus $2.2 \pm 3.2 \mathrm{mg} / \mathrm{dL}$ in those without $\mathrm{MS}(P=$ $0.0001)$. Both biomarkers significantly increased with the number of MS components: Lp-PLA2 levels increased from 
TABLE 3: Correlation coefficients of Lp-PLA2 with demographic variables, lipid factors, blood glucose, blood pressure, hsCRP, and fibrinogen.

\begin{tabular}{|c|c|c|c|c|c|}
\hline \multicolumn{3}{|c|}{ Lp-PLA2 } & \multicolumn{3}{|c|}{ hsCRP } \\
\hline Variable & Correlation coefficient & $P$ & Variable & Correlation coefficient & $P$ \\
\hline BMI & 0.20 & 0.02 & BMI & 0.54 & $<0.0001$ \\
\hline Waist & 0.28 & $<0.001$ & Waist & 0.48 & $<0.0001$ \\
\hline Blood glucose & 0.05 & 0.56 & Blood glucose & 0.17 & $<0.05$ \\
\hline SBP & 0.09 & 0.25 & SBP & 0.23 & $<0.01$ \\
\hline DBP & 0.18 & 0.03 & DBP & 0.15 & 0.07 \\
\hline LDL-C & 0.62 & $<0.001$ & LDL-C & 0.15 & 0.07 \\
\hline HDL-C & -0.45 & $<0.001$ & HDL-C & -0.25 & $<0.01$ \\
\hline Non-HDL-C & 0.66 & $<0.001$ & Non-HDL-C & 0.27 & $<0.001$ \\
\hline hsCRP & -0.04 & 0.67 & Lp-PLA2 & 0.40 & $<0.0001$ \\
\hline Fibrinogen & -0.02 & 0.79 & Fibrinogen & 0.40 & $<0.0001$ \\
\hline Creatinine & 0.32 & $<0.001$ & Creatinine & 0.02 & NS \\
\hline
\end{tabular}

BMI: body mass index; DBP: diastolic blood pressure; HDL-C: high-density lipoprotein cholesterol; hsCRP: high sensitive C-reactive protein; LDL-C: lowdensity lipoprotein cholesterol; Lp-PLA2: lipoprotein-associated phospholipase A2; NS: not significant; SBP: systolic blood pressure.

TABLE 4: Mean levels of hsCRP and Lp-PLA2 by number of metabolic syndrome components according to harmonized criteria ${ }^{1}$ in the total sample.

\begin{tabular}{lccc}
\hline Total $(n=152)$ & $\begin{array}{c}\text { NO MS } \\
(0-2 \mathrm{RF}) \\
(n=95)\end{array}$ & $\begin{array}{c}\text { MS } \\
(n \text { or more RF })\end{array}$ & $P$ \\
\hline $\begin{array}{l}\text { LpPLA2 levels } \\
(\mathrm{nmol} / \mathrm{mL} / \mathrm{min})\end{array}$ & $180 \pm 48$ & $198 \pm 45$ & 0.03 \\
hsCRP $(\mathrm{mg} / \mathrm{L})$ & $2.2 \pm 3.2$ & $4.1 \pm 3.3$ & $<0.0001$ \\
\hline
\end{tabular}

hsCRP: high sensitive C-reactive protein; Lp-PLA2: lipoprotein-associated phospholipase A2; MS: metabolic syndrome; RF: risk factors.

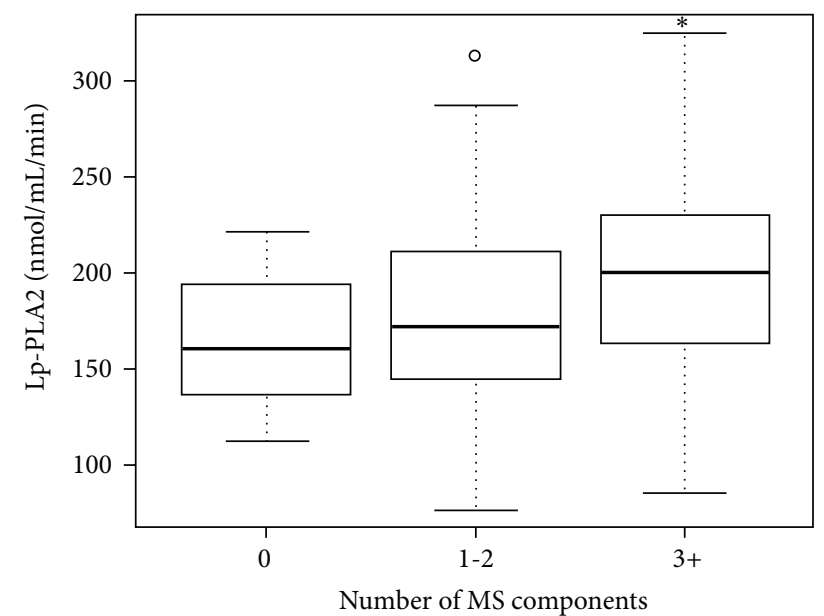

FIGURE 1: Levels of lipoprotein-associated phospholipase A2 by number of metabolic syndrome components. ${ }^{*} P<0.01$ versus 0 MS components. LpPLA2: lipoprotein-associated phospholipase A2; MS: metabolic syndrome.

$163 \mathrm{nmol} / \mathrm{mL} / \mathrm{min}$ to $198 \mathrm{nmol} / \mathrm{mL} / \mathrm{min}(P<0.01)$ and hsCRP increased from $1.5 \mathrm{mg} / \mathrm{L}$ to $4.1 \mathrm{mg} / \mathrm{L}(P=0.04)$ when subjects with 0 and $\geq 3$ components of MS were compared (Figures 1 and 2).

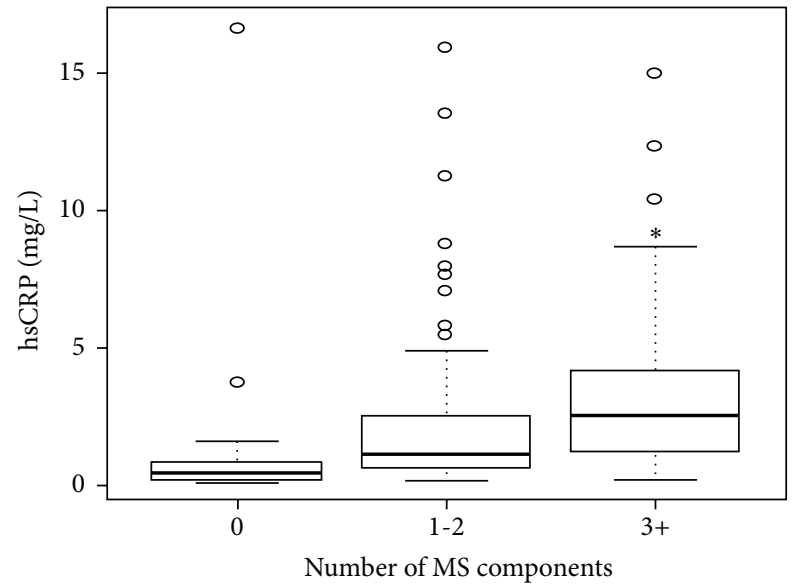

Figure 2: Mean levels of high sensitive C-reactive protein by number of metabolic syndrome components. ${ }^{*} P=0.04$ versus 0 MS components. hsCRP: high sensitivity C-reactive protein; MS: metabolic syndrome.

The results of the logistic regression analysis for determining MS, which included both biomarkers adjusted for age and gender, were as follows: odds ratio (OR) for LpPLA2: 1.02 (confidence interval $[\mathrm{CI}]: 1.00-1.02, P=0.03$ ) and $\mathrm{OR}$ for hsCRP: 2.5 (CI: $1.65-3.80, P<0.0001$ ). Figure 3 shows the ROC curves for both biomarkers adjusted for age and gender: Lp-PLA2, $C$ value $=0.66[0.57-0.74]$ and hsCRP, $C$ value $=$ 0.73 [0.65-0.81]. According to this analysis, although hsCRP shows a better area under the curve, from a statistical point of view, it cannot be shown that it is better than Lp-PLA2, as the CIs of both biomarkers overlap.

\section{Discussion}

In this study, we have demonstrated in a population without atherosclerotic disease that levels of Lp-PLA2 activity and hsCRP are elevated in subjects with MS. Both biomarkers 


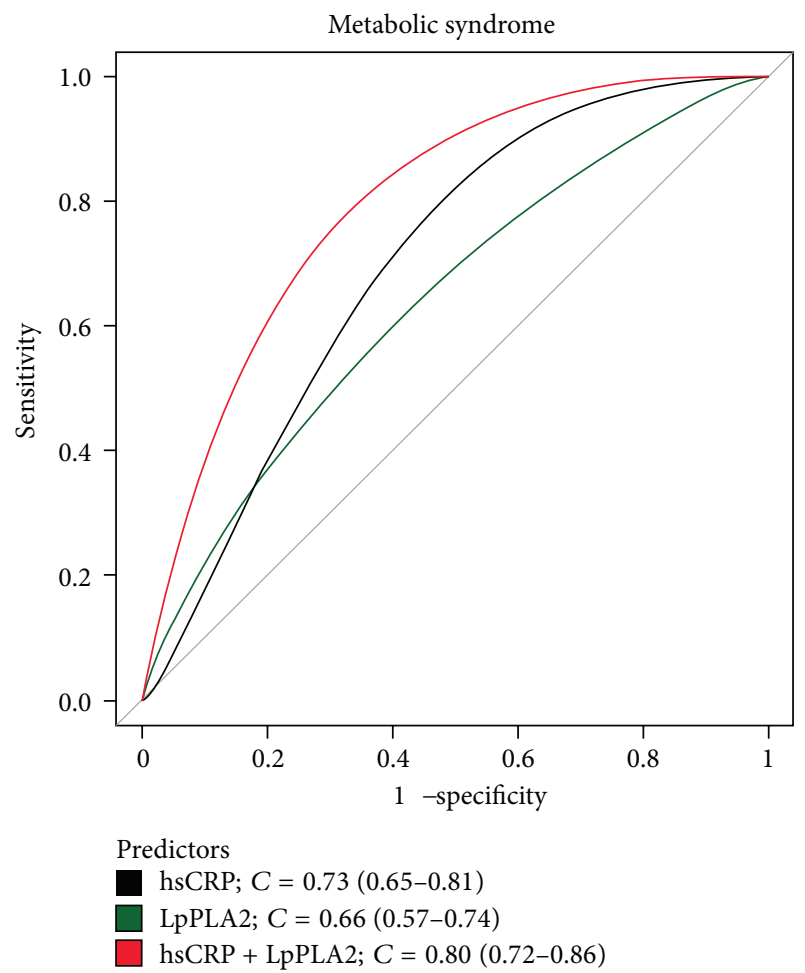

FIGURE 3: ROC curves for lipoprotein-associated phospholipase A2 (Lp-PLA2), high sensitivity C-reactive protein (hsCRP), and both together adjusted by age and gender.

significantly increased with the number of metabolic risk factors, and both were shown to be independent and statistically significant predictors of MS. These results highlight the role of inflammation in MS.

The MS corresponds to a clustering of $\mathrm{CV}$ and metabolic risk factors and inflammation. In our country, MS prevalence is approximately $30 \%$, and it is occurring at younger ages, probably due to the overweight, obesity, and insulin resistance epidemic. Although, the pathophysiologic mechanism involved in MS is not entirely clear, it has been shown that it confers an increased risk for diabetes and atherosclerotic CV disease $[9,15]$.

Inflammation plays a fundamental role in the development of MS. Recent studies have reported that the presence of MS and inflammation (determined by hsCRP) confers more CV risk than the presence of MS alone [16, 17]. High sensitivity C-reactive protein is also a predictor for the development of MS and diabetes [18]. In our country, we have previously reported that subjects with MS and elevated hsCRP have more subclinical atherosclerosis and carotid atherosclerotic plaques [7].

It is well known that atherosclerosis is an inflammatory disease [19]. Therefore, many inflammatory markers related to MS have been investigated in the search of a possible explanation for the elevated CV risk associated with it. The most studied biomarker is hsCRP; however, there are new biomarkers associated with atherosclerotic disease that may be more specific to vascular inflammation. Among them is
Lp-PLA2, an enzyme that acts on oxidized LDL and produces strong inflammatory and oxidative mediators of the intima. Epidemiologic and clinical studies have shown an association between atherosclerotic disease and Lp-PLA2 concentration, in a similar way as reported with hsCRP [9-11, 13].

In this study, we have demonstrated that both biomarkers, Lp-PLA2 and hsCRP, predicted MS with a similar power. Regarding the association of hsCRP with MS, this study confirms the available evidence showing that levels of hsCRP are increased in subjects with MS $[16,17,20]$. Our results also support the association between hsCRP and overall/visceral obesity, HDL-C, non-HDL-C, and glycemia. Similar to the findings of Ballantyne and colleagues [10], we demonstrated that hsCRP is not associated with Lp-PLA2, suggesting that their inflammation pathways are different from a physiologic point of view. Conversely, hsCRP was related to fibrinogen, also an acute phase protein that has a similar mechanism of production by the liver. hsCRP is produced by the liver, primarily by the action of interleukin- 6 , a cytokine that increases in the conditions of visceral obesity and insulin resistance. Based on this evidence, although hsCRP is an important inflammatory marker, it is unspecific.

Activity levels of Lp-PLA2 were also significantly higher in subjects with MS in our study. These findings are similar to those from the Bruneck Study in 2009 [12]. In that study, Tsimikas et al. found significant associations between LpPLA2 and LDL-C, non-HDL-C, HDL-C, and homeostasis model assessment insulin resistance (HOMA-IR), whereas correlations with BMI and waist circumference were weaker. Our results are identical to those reported by these authors regarding lipid factors and obesity. However, we did not find any direct correlation with blood glucose, and we did not measure insulin resistance. Furthermore, Lp-PLA2 was not correlated to BP and smoking in our study, which was seen in the Bruneck Study. Lastly, we found no association between Lp-PLA2 and fibrinogen, which confirms that LpPLA2 inflammatory pathway is not through acute phase reaction.

It is important to analyze the relationship between LpPLA2 and LDL-C and non-HDL-C (atherogenic cholesterol). After adjusting for gender and age, these correlations became even more significant. Our population had an average LDLC level of $128 \mathrm{mg} / \mathrm{dL}$, which suggests that, even at moderate levels of LDL-C, subjects with MS had elevated activity of this enzyme. This result could be because many subjects with MS have primarily small and dense LDL molecules, which are prone to oxidation and therefore they are substrate for LpPLA2. The inflammation produced by Lp-PLA2 is primarily in the vascular level, because lysophosphatidylcholine and oxidized fatty acids, generated after Lp-PLA2 action over oxidized LDL, induce adhesion molecules and other cytokines in the same vascular wall. Thus, it can be hypothesized that, unlike hsCRP (which occurs as a result of inflammation caused by other cytokines from the visceral fat), Lp-PLA2 is directly involved in the inflammatory process, and, subsequently, the atherogenic process of the plaque. Thus, Lp-PLA2 could be a more specific marker of atherosclerotic events in patients with MS. Confirmation of this hypothesis must be demonstrated by studies of $\mathrm{CV}$ events in patients with 
MS, who have these biomarkers measured, and with findings adjusted for all the risk factors associated with hsCRP and LpPLA2.

In this regard, it should be noted that a meta-analysis evaluating the clinical use of hsCRP has shown that this biomarker adds little in the prognosis of CV risk, when adjusting by the risk factors involved in MS [21]. Conversely, the fact that Lp-PLA2 was weakly related to these metabolic factors should preserve its power as a biomarker. However, the latter has not been easy to confirm. The reasons for not having completely cleared Lp-PLA2 prognosis utility are based primarily on the different techniques used for its measurement, which makes it impossible to compare the results of different studies.

In this study, we did not expect to find the same power for hsCRP and Lp-PLA2 as predictors of MS. We expected hsCRP to be a better predictor, given its close association with metabolic risk factors included in MS. However, from a statistical point of view (as demonstrated by the ROC curves), both had a similar level of prediction. These results suggest that Lp-PLA2 is related to MS through other pathways that are not fully known and most likely not associated with the metabolic risk factors. We know that Lp-PLA2 is linked through oxidized LDL, which is elevated in subjects with MS, although it cannot be perceived due to the moderate levels of LDL-C observed in the subjects of our study. The explanation is that Lp-PLA2 is associated with "oxidized" LDL, and, for measuring "oxidized" LDL in the clinic, it required more expensive and sophisticated methods not frequently used in clinical practice.

Since MS is highly prevalent in our population, our results could provide knowledge about nontraditional risk factors that could help to identify which patients with MS must be treated earlier and more aggressively. This is important, because currently there is a pharmacologic inhibitor of LpPLA2 activity, darapladib, which is being investigated in subjects with atherosclerotic disease. In addition, another study is currently investigating the use of a monoclonal antibody against interleukin- 1 for the intervention against high hsCRP. If these studies have positive results, the measurement and targeting of these biomarkers might become important components of clinical management of patients. Finally, it must be noted that we found no studies in literature that compare Lp-PLA2 and hsCRP as predictors of MS in the general population.

Our study has some limitations: (1) it is a cross-sectional study, and hence causality inferences cannot be made; (2) it was done in a small, nonrandom sample of subjects; (3) we did not include HOMA-IR, which could explain the relationship of insulin resistance with MS and inflammatory markers; (4) finally, we only determined Lp-PLA2 activity instead of mass. This decision was subject to the availability of the laboratory determination in our country. However, currently, it is the most used technique, and it has shown the best correlation with MS [13].

The strengths of the study include the following: (1) the recruitment of subjects was performed prospectively; (2) subjects with lipid-lowering therapy and women with hormone replacement therapy were excluded, among others;
(3) this study has validated the measurement of Lp-PLA2 in our country.

In conclusion, Lp-PLA2 and hsCRP are good determinants of MS. Prospective studies must confirm which of the markers or if both markers are causally related to the atherosclerotic consequences observed in subjects with MS.

\section{Conflict of Interests}

The authors declare that there is no conflict of interests regarding the publication of this paper.

\section{Authors' Contribution}

Mónica Acevedo and Paola Varleta contributed equally to this work.

\section{Acknowledgment}

The authors acknowledge Emily Donovan for the editorial assistance.

\section{References}

[1] K. G. M. M. Alberti, R. H. Eckel, S. M. Grundy et al., "Harmonizing the metabolic syndrome: a joint interim statement of the International Diabetes Federation Task Force on Epidemiology and Prevention; National Heart, Lung, and Blood Institute; American Heart Association; World Heart Federation; International Atherosclerosis Society; and International Association for the Study of Obesity," Circulation, vol. 120, no. 16, pp. 1640$1645,2009$.

[2] P. Dandona, A. Aljada, A. Chaudhuri, P. Mohanty, and R. Garg, "Metabolic syndrome: a comprehensive perspective based on interactions between obesity, diabetes, and inflammation," Circulation, vol. 111, no. 11, pp. 1448-1454, 2005.

[3] P. Libby, P. M. Ridker, and A. Maseri, "Inflammation and atherosclerosis," Circulation, vol. 105, no. 9, pp. 1135-1143, 2002.

[4] H. S. Park, J. Y. Park, and R. Yu, "Relationship of obesity and visceral adiposity with serum concentrations of CRP, TNF- $\alpha$ and IL-6," Diabetes Research and Clinical Practice, vol. 69, no. 1, pp. 29-35, 2005.

[5] P. M. Ridker, E. Danielson, F. A. Fonseca et al., "Reduction in Creactive protein and LDL cholesterol and cardiovascular event rates after initiation of rosuvastatin: a prospective study of the JUPITER trial," The Lancet, vol. 373, no. 9670, pp. 1175-1182, 2009.

[6] P. M. Ridker, D. A. Morrow, L. M. Rose, N. Rifai, C. P. Cannon, and E. Braunwald, "Relative efficacy of atorvastatin $80 \mathrm{mg}$ and pravastatin $40 \mathrm{mg}$ in achieving the dual goals of low-density lipoprotein cholesterol $<70 \mathrm{mg} / \mathrm{dl}$ and C-reactive protein <2 mg/L: an analysis of the PROVE-IT TIMI-22 trial," Journal of the American College of Cardiology, vol. 45, no. 10, pp. 1644-1648, 2005.

[7] M. Acevedo, P. Arnaiz, R. Corbalán et al., "Modificación del grosor intima-media carotídeo según factores de riesgo clásicos y sindrome metabólico con o sin inflamación," Revista Chilena de Cardiología, vol. 28, pp. 337-348, 2009. 
[8] P. P. Toth, P. A. McCullough, M. S. Wegner, and K. J. Colley, "Lipoprotein-associated phospholipase $\mathrm{A}_{2}$ : role in atherosclerosis and utility as a cardiovascular biomarker," Expert Review of Cardiovascular Therapy, vol. 8, no. 3, pp. 425-438, 2010.

[9] The Lp-PLA2 Studies Collaboration, "Lipoprotein-associated phospholipase $\mathrm{A}_{2}$ and risk of coronary disease, stroke, and mortality: collaborative analysis of 32 prospective studies," The Lancet, vol. 375, no. 9725, pp. 1536-1544, 2010.

[10] C. M. Ballantyne, R. C. Hoogeveen, H. Bang et al., "Lipoproteinassociated phospholipase $\mathrm{A}_{2}$, high-sensitivity C-reactive protein, and risk for incident coronary heart disease in middle-aged men and women in the Atherosclerosis Risk in Communities (ARIC) study," Circulation, vol. 109, no. 7, pp. 837-842, 2004.

[11] C. J. Packard, D. S. J. O’Reilly, M. J. Caslake et al., "Lipoproteinassociated phospholipase A2 as an independent predictor of coronary heart disease," The New England Journal of Medicine, vol. 343, no. 16, pp. 1148-1155, 2000.

[12] S. Tsimikas, J. Willeit, M. Knoflach et al., "Lipoproteinassociated phospholipase A2 activity, ferritin levels, metabolic syndrome, and 10-year cardiovascular and non-cardiovascular mortality: Results from the Bruneck study," European Heart Journal, vol. 30, no. 1, pp. 107-115, 2009.

[13] M. Persson, J.-A. Nilsson, J. J. Nelson, B. Hedblad, and G. Berglund, "The epidemiology of Lp-PLA 2 : distribution and correlation with cardiovascular risk factors in a populationbased cohort," Atherosclerosis, vol. 190, no. 2, pp. 388-396, 2007.

[14] A. V. Chobanian, G. L. Bakris, H. R. Black et al., "The seventh report of the Joint National Committee on prevention, detection, evaluation, and treatment of high blood pressure: the JNC 7 report," Journal of the American Medical Association, vol. 289, no. 19, pp. 2560-2572, 2003.

[15] S. M. Grundy, J. I. Cleeman, S. R. Daniels et al., "Diagnosis and management of the metabolic syndrome: an American Heart Association/National Heart, Lung, and Blood Institute scientific statement," Circulation, vol. 112, no. 17, pp. 2735-2752, 2005.

[16] P. M. Ridker, J. E. Buring, N. R. Cook, and N. Rifai, "Creactive protein, the metabolic syndrome, and risk of incident cardiovascular events: an 8-year follow-up of 14719 initially healthy American women," Circulation, vol. 107, no. 3, pp. 391397, 2003.

[17] P. M. Ridker, P. W. F. Wilson, and S. M. Grundy, "Should C-reactive protein be added to metabolic syndrome and to assessment of global cardiovascular risk?" Circulation, vol. 109, no. 23, pp. 2818-2825, 2004.

[18] T. S. Han, N. Sattar, K. Williams, C. Gonzalez-Villalpando, M. E. J. Lean, and S. M. Haffner, "Prospective study of C-reactive protein in relation to the development of diabetes and metabolic syndrome in the Mexico City diabetes study," Diabetes Care, vol. 25, no. 11, pp. 2016-2021, 2002.

[19] R. Ross, "Atherosclerosis-an inflammatory disease," The New England Journal of Medicine, vol. 340, no. 2, pp. 115-126, 1999.

[20] D. Aronson, P. Bartha, O. Zinder et al., "Obesity is the major determinant of elevated C-reactive protein in subjects with the metabolic syndrome," International Journal of Obesity, vol. 28, no. 5, pp. 674-679, 2004.

[21] S. Tsimikas, J. T. Willerson, and P. M. Ridker, "C-reactive protein and other emerging blood biomarkers to optimize risk stratification of vulnerable patients," Journal of the American College of Cardiology, vol. 47, no. 8, supplement, pp. C19-C31, 2006. 


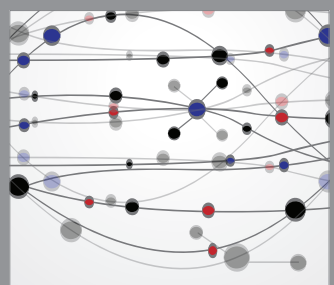

The Scientific World Journal
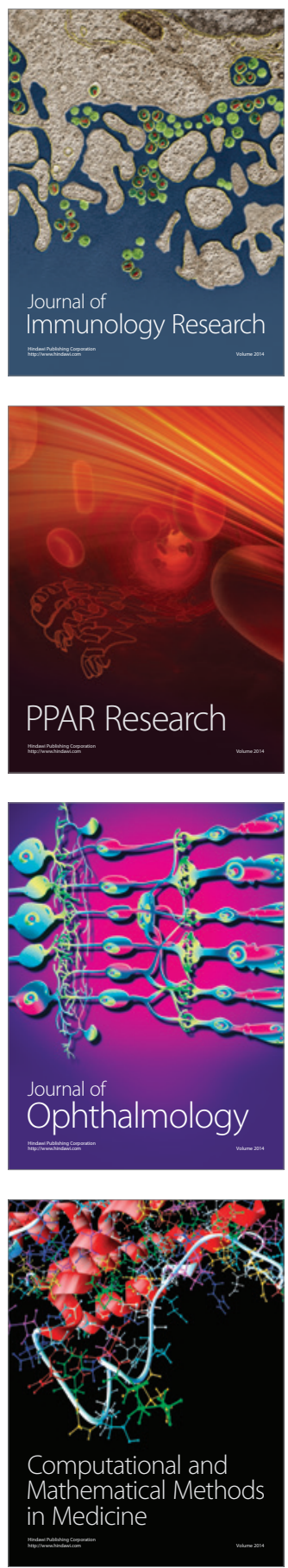

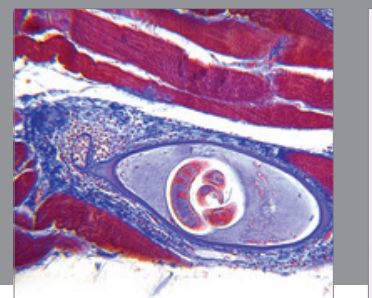

Gastroenterology

Research and Practice
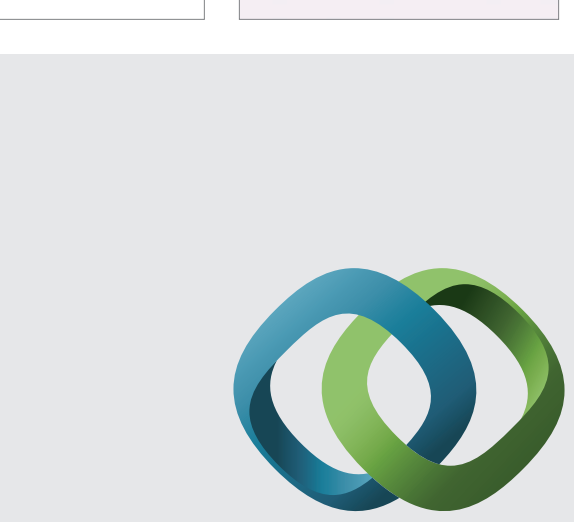

\section{Hindawi}

Submit your manuscripts at

http://www.hindawi.com
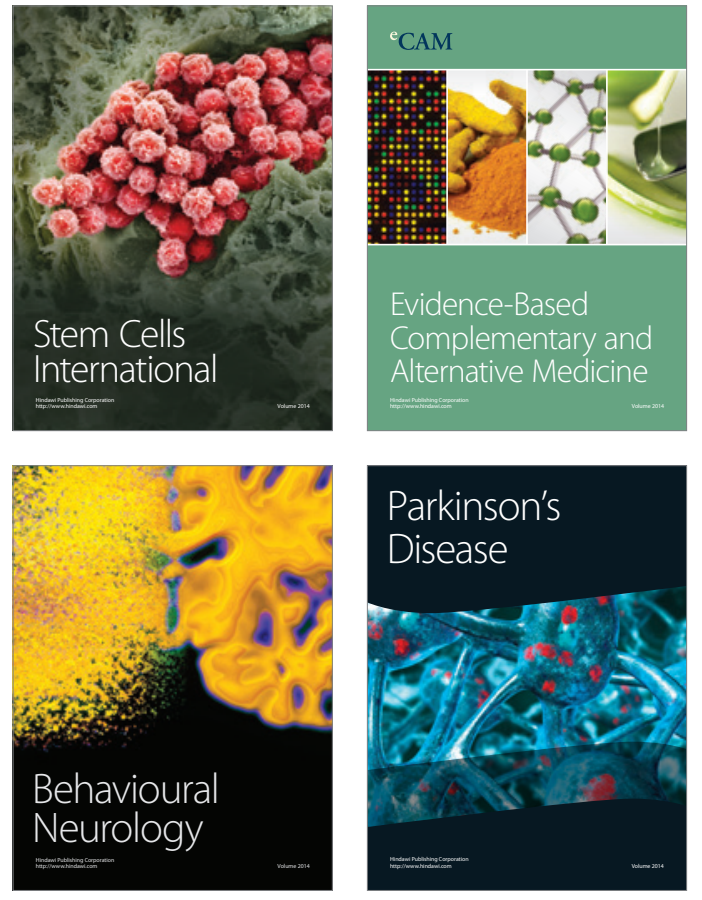
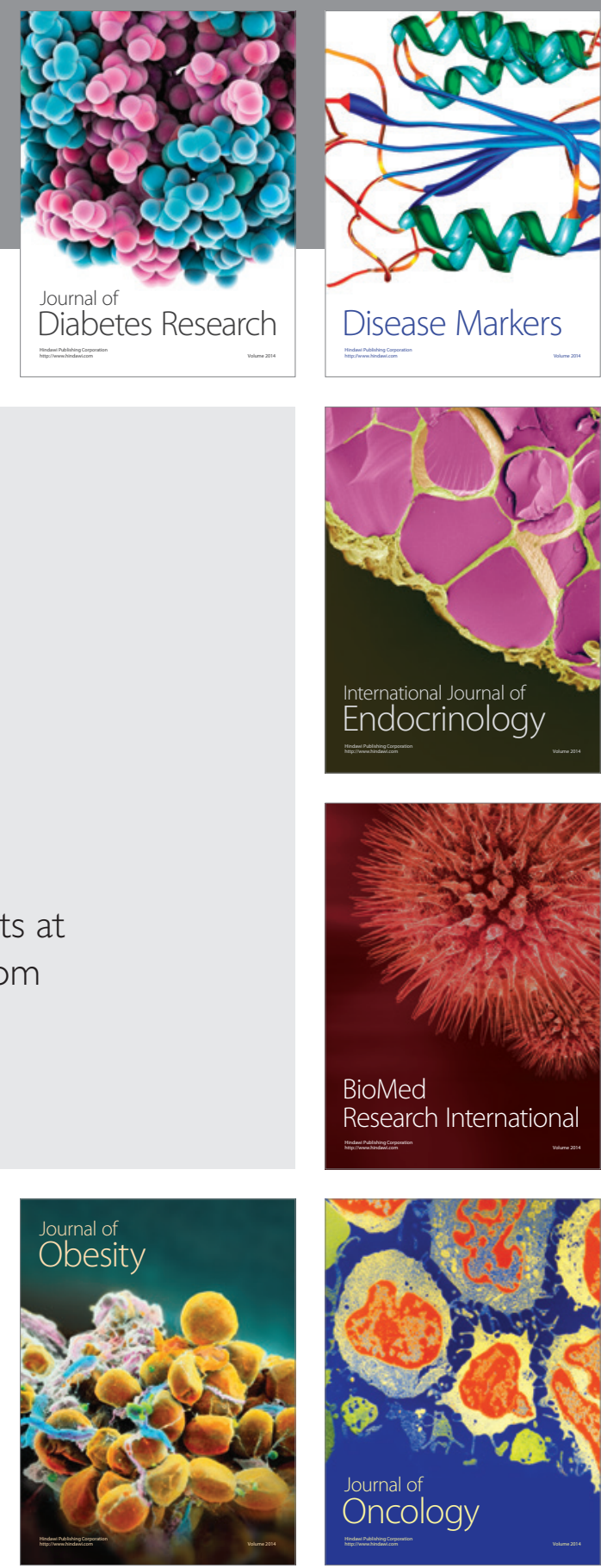

Disease Markers
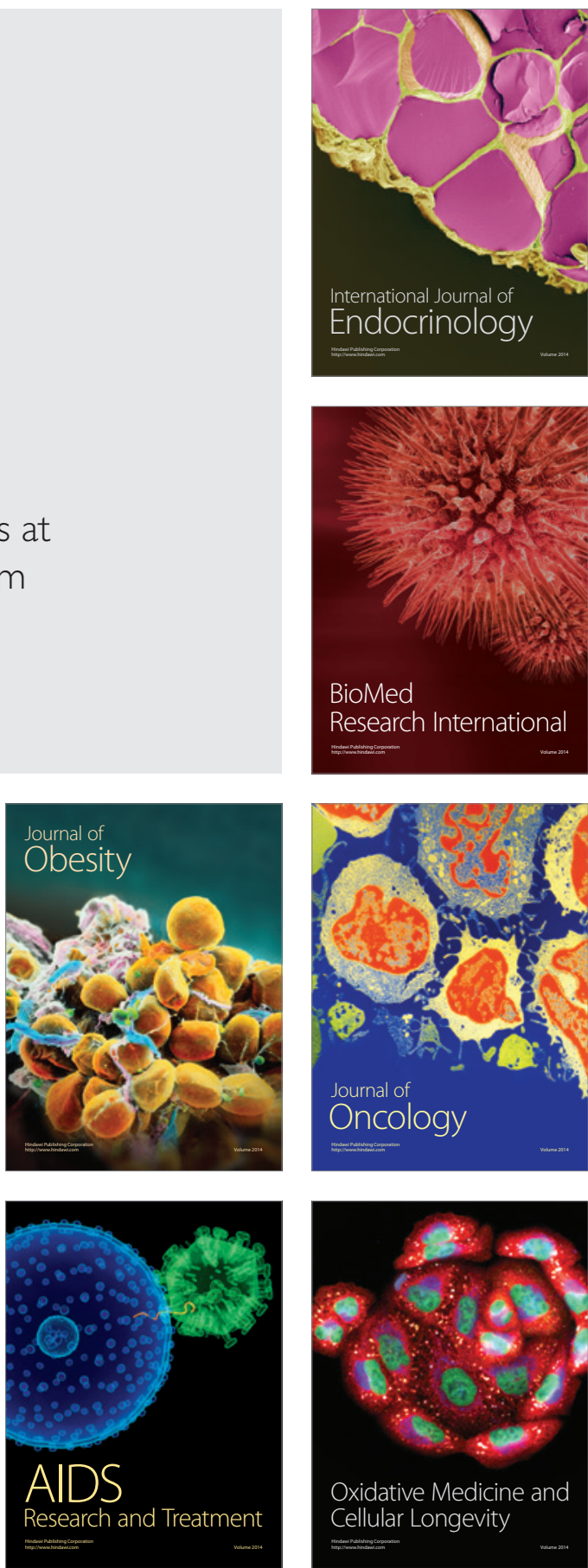